American Journal of Pharmacology and Toxicology 6 (2): 59-67, 2011

ISSN 1557-4962

(C) 2011 Science Publications

\title{
Effects of Melatonin in Prevention of Neuropathy in STZ-Induced Diabetic Rats
}

\author{
${ }^{1,2}$ Nasser Zangiabadi, ${ }^{1}$ Vahid Sheibani, ${ }^{1}$ Majid Asadi-Shekaari, \\ ${ }^{1}$ Mohammad Shabani, ${ }^{1}$ Mandana Jafari, ${ }^{2}$ Ali Reza Asadi, \\ ${ }^{1}$ Haleh Tajadini and ${ }^{1}$ Morteza Jarahi \\ ${ }^{1}$ Neuroscience Research Center, \\ Kerman University of Medical Sciences, Kerman, Iran \\ ${ }^{2}$ Afzal Research Center, Kerman, Iran
}

\begin{abstract}
Problem statement: Diabetes mellitus occurs mainly with chronic polyneuropathy, and oxidative stress plays an important role in emergence of most neurologic and behavioral changes in diabetic patients. Many studies have focused on the beneficial effects of various antioxidants such as melatonin on diabetic neuropathy. The aim of this study is to evaluate the effect of melatonin in prevention of neuropathy in Streptozotocin-induced diabetic rats. After prescribing Streptozotocin (STZ), treatment rats received melatonin $\left(10 \mathrm{mg} \mathrm{kg} \mathrm{day}{ }^{-1}\right)$ or DMSO for a period of 6 weeks. Approach: At the end of the sixth week, non diabetic control group, diabetic control group (sham) and treated rats were examined by thermal pain response tests (hot plate and tail flick). The horizontal and vertical activities of rats were measured in an open field test. After that, Motor Nerve Conduction Velocity (MNCV) of sciatic-tibial nerve recorded. Also, to study morphological alterations resulting from diabetic neuropathy of sciatic nerve, Myelinated Fiber Diameter (MFD), Axon Diameter (AD) and Myelin Sheath Diameter (MSD) were evaluated by light microscope. Results: According to hot plate results, response time to thermal pain at the end of sixth week in sham group showed a significant decrease in comparison with the control group $(\mathrm{p}<0.01)$. In hot plate test, although melatonin approximated to the response time to control group, the significant difference was not observed among melatonin receivers and other groups. In the open field test, Total Distance Moved (TDM) and mobility duration showed significant decrease in sham and DMSO groups in comparison to the control and melatonin groups. Diabetic rats treated with melatonin showed significant increase in MNCV compared to sham and DMSO groups $(\mathrm{p}<0.05)$. In morphological study, pretreatment with Melatonin significantly reversed sciatic nerve diameters (MFD, AD, and MSD) reduction in diabetic rats. Electron microscopy showed myelin splitting and myelin sheath infolding in diabetic control group compare to non diabetic group. Conclusion: This study showed that melatonin can decrease the destructive progress of diabetes and causes neuroprotection against damages resulting from STZinduced hyperglycemia.
\end{abstract}

Key words: Sciatic nerve, Axon Diameter (AD), Myelinated Fiber Diameter (MFD), Motor Nerve Conduction Velocity (MNCV), diabetic rats, Reactive Oxygen Species (ROS)

\section{INTRODUCTION}

Hyperglycemia in diabetic patients as the main factor of diabetic neuropathy induces oxidative stress through various cellular pathways such as increasing aldose reductase activity (Srivastava et al., 2005), increasing glycation end-products (Sugimoto et al., 2008) and altering protein kinas $\mathrm{C}$ activity (Yamagishi et al., 2008). Longstanding hyperglycemia through producing a large amount of Reactive Oxygen Species (ROS) can damage mitochondrial DNA in dorsal root ganglia leading to peripheral nerves dysfunction (Schmeichel et al., 2003). Several studies have proposed that oxidative stress is one of the major factors impairing sensory nerves and dorsal root ganglia (Arora et al., 2008; Sharma et al., 2009). Today many studies have focused on the beneficial effects of various antioxidants such as melatonin on diabetic neuropathy (Zangiabadi et al.,

Corresponding Author: Nasser Zangiabadi, Neuroscience Research Center, Kerman University of Medical Sciences, Kerman, Iran, P.O. Box: 76198-13159 Tel: +98-341-226-4198/+98-913-3978116 
2007; Negi et al., 2010; Srinivasan et al., 2010). Melatonin is one of the tryptophan derivatives that is mainly synthesized in the pineal gland and also in retina, intestine, ovary, testis and bone marrow (Pevet et al., 1980; Gutierrez-Cuesta et al., 2007; Geoffriau et al., 1998). The physiologic importance of melatonin has not been recognized yet and its intervention in circadian rhythms has already been proposed as the most important function of this hormone. Melatonin in addition to physiologic functions such as controlling day-night rhythm, inducing sleep, seasonal regulation of reproduction and increasing immune system activity has been known as a powerful biologic antioxidant in neutralizing free radicals (Tan et al., 2007; Hardeland and Pandi-Perumal, 2005). Moreover, some psychopharmacological effects like sedative, antiepileptic and anti-anxiety effects have been proposed for melatonin (Geoffriau et al., 1998). It has been found in some behavioral studies that melatonin has a prolonged and severe antinociceptive effect in neuropathic pains via interaction with opioidergic and GABAergic system receptors (Golombek et al., 1996; Mantovani et al., 2003). Although, several studies have mentioned melatonin role in the treatment of neuropathy due to diabetes (Negi et al., 2010; Srinivasan et al., 2010; Arreola-Espino et al., 2007), there are few studies about its effect on prevention of diabetic neuropathy. Therefore, the present study was designed to investigate the protective effects of melatonin in prevention of neuropathy in Streptozotocin -induced diabetic rats.

\section{MATERIALS AND METHODS}

This study was performed on Wistar rats weighing 200-250gr. The care of laboratory animals followed the guiding principles for care and use of laboratory animals of the Neuroscience Research Center of Kerman Medical University and the study protocol was approved by the animal ethics committee of this institution (Code:EC/KNRC/88-15).

Animals were kept in standard conditions, temperature of $22 \pm 2^{\circ} \mathrm{Cand} 12$-h dark-light cycle. They had free access to food and water. These rats were divided into four groups: First group consisted of control animals. The second, third and fourth groups consisted of diabetic animals. The second group was as diabetic control group (sham). The third and fourth groups treated with melatonin $\left(10 \mathrm{mg} \mathrm{kg} \mathrm{day}{ }^{-1}\right.$, i.p.) and DMSO, at least 8 rats in every group. Diabetes was induced by intra-peritoneal injection of $45 \mathrm{mg} \mathrm{kg}^{-1}$ Streptozotocin. In order to confirm diabetes, three days after Streptozotocin injection blood glucose was measured using glocometer instrument (Accu-checkactive, ROCHE, Germany) and animals with blood glucose of over $200 \mathrm{mg} \mathrm{dL}{ }^{-1}$ were considered as diabetics. In order to investigate the effect of melatonin in prevention of neuropathy, melatonin injection $(10 \mathrm{mg}$ $\mathrm{kg}$ day $^{-1}$ ) started 4 days after STZ injection and confirmation of diabetes and continued for 6 weeks.

Hot plate test: In Hot Plate Test, pain sensitivity was evaluated by using an apparatus (LE710 model, Lsi LETICA, Spain) that contained a plate with the diameter of $19 \mathrm{~cm}$ and a Plexiglas wall with height of $30 \mathrm{~cm}$. Plate temperature was adjusted to $52 \pm 2^{\circ} \mathrm{C}$. Response time to thermal pain was considered as the time between test onset and licking front paw or jumping (maximum cut off was considered $60 \mathrm{sec}$ ); (Sharma et al., 2007).

Tail flick: Tail flick test is one of the standard tests for measuring analgesia amount. In this test, thermal light was shown to the end of tail of rats with intensity of 5 using tail flick machine (made by Spanish Lsi LETICA, model LE7406) and tail flick latency was measured from the light emission to take the tail (on second). To prevent tissue damage, maximum light mission to the tail was considered $10 \mathrm{sec}$. For each animal rat, tail flick latency was measured for three times and the average was reported as tail flick latency. Between every test, rats were left free for half an hour (Liepinsh et al., 2009).

Open field test: This test was used to investigate the effect of diabetic neuropathy on explorative behavior of diabetes-induced rats and the protective effect of melatonin. Explorative behavior of rats was studied by a video Track System (TSE) in a $45 \times 45 \times 45 \mathrm{~cm}$ black wood box. At the end of the 6th week following diabetes induction, animals were placed in the center of field and their explorative behavior including the distance in center and border areas and time spent in center and border areas as well as the frequency of grooming and rearing were detected for $5 \mathrm{~min}$ (Shabani et al., 2011).

Electrophysiological evaluation: Six weeks after initiation of hyperglycemia, animals were anesthetized by injection of Ketamin/Xylozine solution (50/20 mg $\mathrm{kg}^{-1}$, i.p). The environment temperature was maintained at $25 \pm 1^{\circ}$ Cduring all stages of study. After shaving the animals' back, a small incision was made in right sciatic notch and ankle. Then, by bi-polar electrodes, proximal part of sciatic notch and distal part of ankle were stimulated and after stimulation, Motor Nerve Conductivity Velocity (MNCV) of sciatic-tibial was recorded (powerlab/ML856). Immediately after each 
stimulation, action potential of first interosseous muscle of the hind paw was recorded by uni-polar electrodes. The obtained records are biphasic responses with a primary M-wave produced due to the stimulation of motor fibers (Kumar et al., 2007; Saini et al., 2007). MNCV was calculated by dividing the distance between the two stimulated sites $(\mathrm{mm})$ by the difference between proximal and distal latencies (ms).

Morphological study of sciatic nerve: For morphometric evaluation, sciatic nerve was isolated and divided into $2 \mathrm{~mm}$ segments. They were fixed immediately with glutaraldehyde solution $2.0 \%$ in 0.1 M Phosphate Buffer Solution (PBS) for $24 \mathrm{~h}$. The specimens were washed with PBS for 3 times and post fixed for $1.5 \mathrm{~h}$ in $1 \%$ tetroxide osmium and dehydrated in graded concentration of ethanol and finally embedded in Epoxy resin. Semithin sections (350 nm) were stained with $1 \%$ toluidin blue and examined by light microscopy. Ten fields of transverse sections were morphometrically analyzed by computerized image analysis system (Motic Images China e-kup Co., Ltd). MFD, AD and MSD were measured for each section.

For ultrastructural study, ultrathin sections (80 $\mathrm{nm}$ ) were stained with $1 \%$ uranyl acetae and $2 \%$ lead citrate before viewing in Philips TEM300 (Philips, Eindhoven, Netherlands).

Statistical analysis: Data are presented as mean \pm SEM. One-way ANOVA was used in order to determine statistical difference and in the case of significant difference, Tukey test was used to determine the difference among the groups. LSD test was used in morphological study and $\mathrm{p}<0.05$ was considered as statistical significant level.

\section{RESULTS}

Metabolic characteristics: Three days after STZ injection, Plasma glucose showed significant increase in diabetic groups as compared to the control group (Control: $106.2 \pm 4.1 \mathrm{mg} \mathrm{dL}^{-1}$, Diabetic rats: $318.9 \pm 10$, $\mathrm{p}<0.001)$. There was no significant difference among groups in regard to the primary weight. Weight gain of diabetic rats at the end of the 7th week was significantly less than that of the control group. That is, in the control group weight had increased at the end of the 7 th week compared to the 1st week, while in diabetic groups of melatonin and DMSO it had decreased (Table 1).

The protective effect of melatonin in response to thermal pain: According to the results of hot plate test, response time to thermal pain in the 7 th week following diabetes induction, showed significant decrease in sham group as compared to the control $(\mathrm{p}<0.001)$, melatonin $(\mathrm{p}<0.05)$ and DMSO groups $(\mathrm{p}<0.001)$. Although in melatonin group, response time to thermal pain showed significant increase $(\mathrm{p}<0.05)$ compared to the sham group and was close to that in the control group, it was significantly lower $(\mathrm{p}<0.05)$ in comparison to the control group. It seemed that in this test, melatonin solvent (DMSO) has better protective effects than melatonin in response to thermal pain. In evaluation of response to thermal pain, there was no significant difference among groups in tail flick test (Fig. 1).

The effect of melatonin on explorative behavior in open field test: In open field test performed to find the effect of diabetic neuropathy and melatonin treatment on rats' explorative behavior, frequency of rearing and grooming in all diabetic groups (melatonin, sham and DMSO) showed significant decrease compared to the control group $(p<0.05)$. The total distance in central and border areas (TDM) and mobility duration showed significant decrease $(\mathrm{p}<0.05)$ in diabetic sham and DMSO groups in comparison to the control and melatonin groups, while there was no significant difference between melatonin and control groups in this regard. There was no significant difference between the control group and none of the diabetic groups of sham, DMSO and melatonin in regard to the time spent in central and border areas and also immobility duration and mobility speed in central and border areas (Fig. 2).

The effects of melatonin on MNCV in diabetic rats: $v$ At the end of the 7th week, MNCV showed significant decrease in diabetic groups of sham and DMSO compared to the control group $(\mathrm{p}<0.05)$. Diabetic rats treated with melatonin for 6 weeks had no significant difference with the control group in $\mathrm{MNCV}$, while they showed significant increase in $\mathrm{MNCV}$ compared to sham and DMSO groups ( $\mathrm{p}<0.05)$ (Fig. 3).

Morphometry: Light microscopy study of sciatic nerves sections showed that normal structure and morphology of myelinated fibers in control group. In Sham and DMSO groups, some abnormalities including increased numbers of mast cells, edema and myelin sheath splitting were seen (Fig. 4). Increasing in number of abnormal myelinated fibers was observed in Sham and vehicle groups. Pretreatment of rats with Melatonin prevented all of these abnormalities in high extent. The mean of MFD and AD were 9.75 \pm 0.45 $(\mu \mathrm{m})$ and $5.11 \pm 0.34,8.67 \pm 0.3$ and $4.2 \pm 0.5,8.25 \pm 0.41$ and $3.73 \pm 0.22,8.15 \pm 0.19$ and $4.09 \pm 0.15$ in Control, Melatonin, DMO and Sham respectively. 
Am. J. Pharm. \& Toxicol., 6 (2): 59-67, 2011

Table 1: Body weight and blood glucose levels of all groups

\begin{tabular}{|c|c|c|c|}
\hline \multirow[b]{2}{*}{ Animal group } & \multicolumn{2}{|l|}{ Body weight (g) } & \multirow{2}{*}{$\begin{array}{l}\text { Blood glucose }\left(\mathrm{mg} \mathrm{dL}^{-1}\right) \\
\text { Before sacrifice }\end{array}$} \\
\hline & Before STZ injection & End experiment & \\
\hline Control (8) & $265 \pm 4.22$ & $286.25 \pm 5.32$ & $106.25 \pm 4.16$ \\
\hline Sham (9) & $261.25 \pm 2.95$ & $220 \pm 5.01 * * * *$ & $306.25 \pm 19.45 * * * *$ \\
\hline DMSO (8) & $269.7 \pm 5.3$ & $238.5 \pm 5.6 * * * *$ & $365 \pm 29.8 * * * *$ \\
\hline Melatonin (11) & $265 \pm 4.2$ & $246.2 \pm 4.8 * * * \dagger$ & $270.7 \pm 25.7 * * * *$ \\
\hline
\end{tabular}

Data are the mean \pm SEM $^{* * * *}: \mathrm{p}<0.001,{ }^{, * * * * *} \mathrm{p}<0.0001$, compared with the control group; ${ }^{\dagger}: \mathrm{p}<0.05$, compared with the sham group

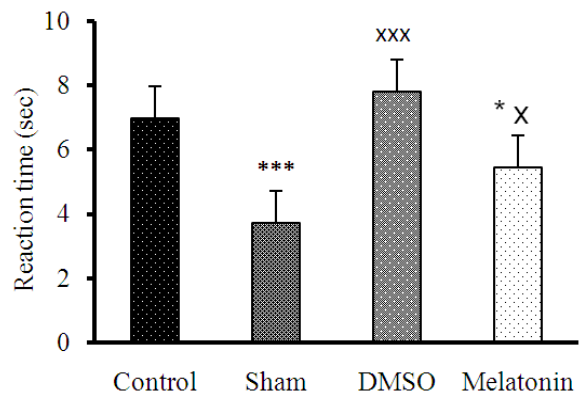

(a)

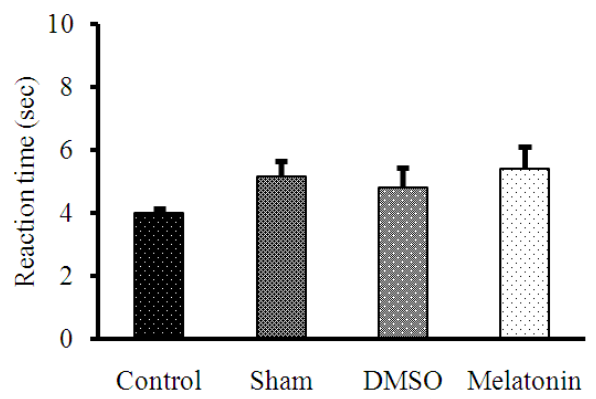

(b)

Fig. 1: Effect of Melatonin (10 $\mathrm{mg} \mathrm{kg} \mathrm{day}^{-1}$, ip, for 6 weeks) on the pain threshold values in streptozotocin-injected diabetic rats subjected to hot plate (A) and tail flick (B). ${ }^{*} \mathrm{P}<0.05,{ }^{* * *} \mathrm{P}<0.001$ as compared to control group, ${ }^{\mathrm{x}} \mathrm{P}<0.05,{ }^{\mathrm{xx}} \mathrm{P}<0.001$ as compared to Sham group. There are no significance effects between DMSO and melatonin -treated diabetic groups. Values are expressed as mean \pm SEM. ( $\mathrm{n}=8$ rats in each group)

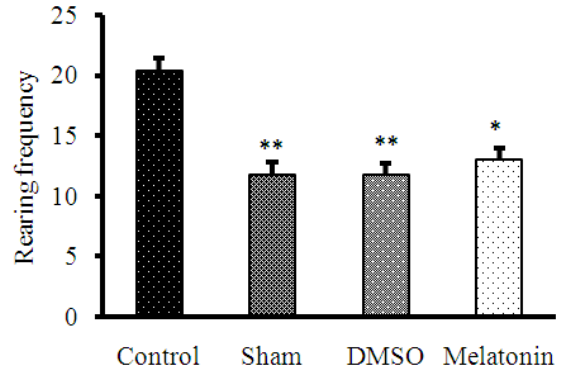

(a)

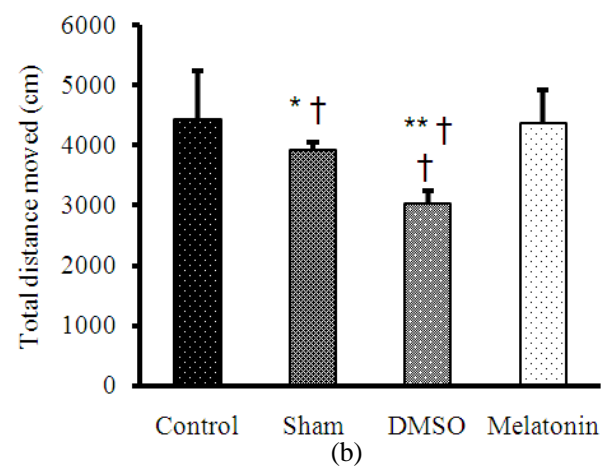

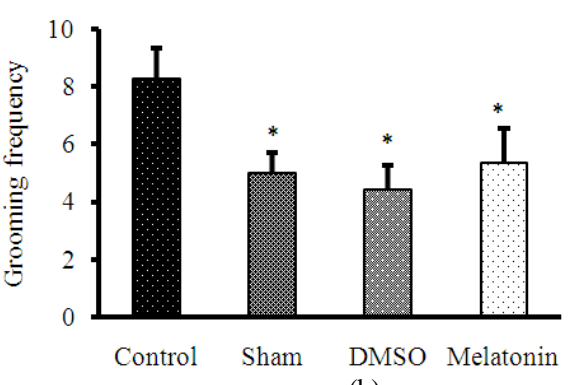

(b)

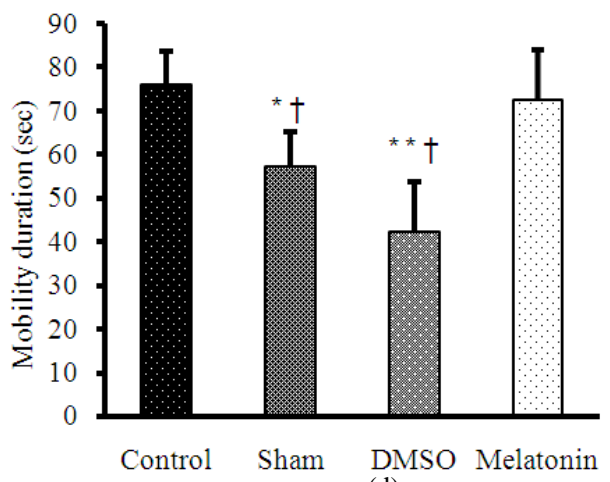

(d)

Fig. 2: Effect of Melatonin on explorative behavior of rats in open field test. Rearing frequency (a), Grooming frequency (b) ,TDM (c) and Mobility duration (d), ${ }^{*} \mathrm{p}<0.05,{ }^{* *} \mathrm{p}<0.01$ compared with the control group, ${ }^{\dagger}<<0.05,{ }^{\dagger} p<0.01$, compared with the Melatonin group. Values are expressed as mean \pm SEM. ( $n=8$ rats in each group) 
Am. J. Pharm. \& Toxicol., 6 (2): 59-67, 2011

Table 2: The effect of Melatonin on histomorphometric parameters of rat sciatic nerve

\begin{tabular}{lllll}
\hline Groups & $\mathrm{N}$ & MFD $(\mu \mathrm{m})$ & AD $(\mu \mathrm{m})$ & MSD $(\mu \mathrm{m})$ \\
\hline Control & 4 & $9.75 \pm 0.45$ & $5.11 \pm 0.34$ & $4.64 \pm 0.35$ \\
Sham & 4 & $8.15 \pm 0.19^{*}$ & $4.1 \pm 0.15^{*}$ & $4.06 \pm 0.23$ \\
DMSO & 4 & $8.25 \pm 0.41^{*}$ & $3.73 \pm 0.22^{*}$ & $4.52 \pm 0.33$ \\
Melatonin & 5 & $8.67 \pm 0.3$ & $4.2 \pm 0.5$ & $4.65 \pm 0.30$ \\
\hline
\end{tabular}

$\mathrm{N}$ : number of animals. MFD: Myelinated Fiber Diameter, AD: Axon Diameter, MSD: Myelin Sheath Diameter. Data are presented as Mean \pm SEM. " $\mathrm{p}<0.05$ Vs. control

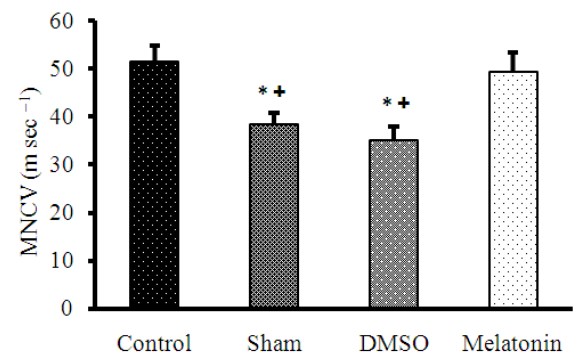

Fig. 3: Effect of melatonin on motor nerve conductive velocity (MNCV) in rats. Data are the mean \pm SEM. ${ }^{*}: \mathrm{p}<0.05$ compared with the control group; $t \mathrm{p}<0.05$, compared ith the date extract group
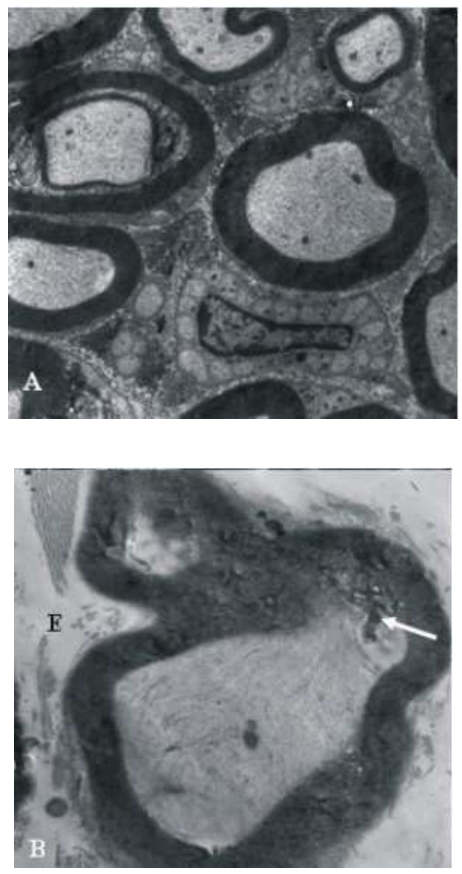

Fig. 4: Electron micrograph of rat sciatic nerves. (a) Control group: Myelinated fiber with normal structure and morphology. Some unmylinated fibers are seen in bottom the picture. (b) Sham group: Myelinated fiber shows myelin infolding and splitting (white arrow). Edema (E) is clear in the field. Axonal degeneration was revealed with neurofilament disintegration. A×8900 B×11500
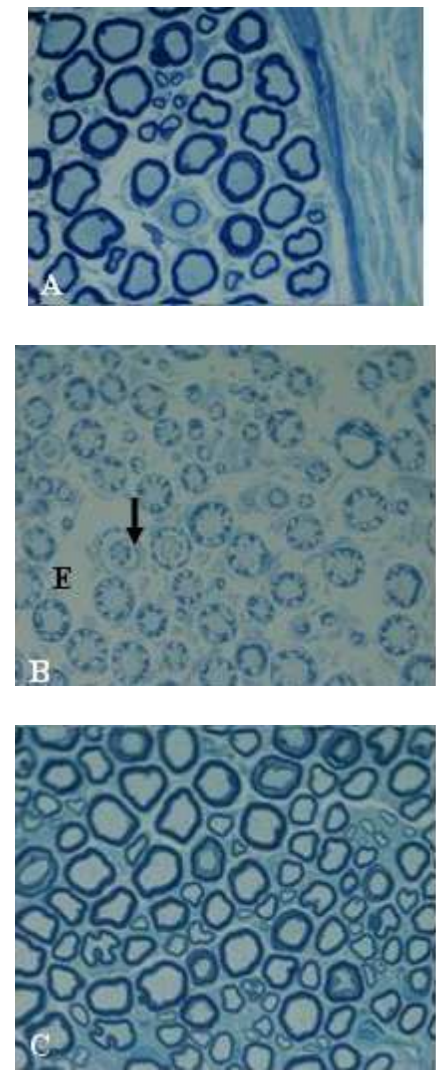

Fig. 5: Light micrograph of transverse semi-thin sections of rat sciatic nerves. (a): Control group, myelinated nerve fibers are in normal structure and morphology. (b): Sham group, nerves fibers show some abnormalitities such as myelin splitting (black arrow), and Edema (E, c): The DFE-treated group, the proportion of nerve fibers with abnormalities was reduced. $\times 1000$. Toluidin blue staining

According to the data, MFD and AD of DMSO and Sham groups were decreased comparison to control group. Pretreatment with Melatonin for 6 weeks significantly reversed each diameter reduction in diabetic rats (Table 2).

Electron microscopy: Ultrastructural examination of sciatic nerves confirmed the light microscopy findings 
(Fig. 5). In addition, some evidences of axonal degeneration such as mitochondrial swelling and disintegration of neurofilaments were observed under transmission electron microscope. Degenerative, reactive and proliferative of Schwann cells were not observed in any groups.

\section{DISCUSSION}

It was observed in the present study that intraperitoneal injection of $10 \mathrm{mg} \mathrm{kg}^{-1}$ melatonin has protective effects against peripheral nerves injury due to diabetic neuropathy. Decrease of motor nerve conductivity velocity in STZ-induced diabetic rats is an evidence of diabetic neuropathy development in these rats. In the present study, we observed $25.6 \%$ defect in MNCV at the 7 th week following diabetes induction in the sham group compared to the control group. While, melatonin after diabetes induction prevented diabetes destructive effects on nerve conductivity in sciatic-tibia motor nerve.

Peripheral neuropathy is one of the major complains in both types I and II diabetic patients and it is associated with several problems such as cardiovascular defects, retinopathy and muscular pain or weakness (Yagihashi et al., 2007; Sima, 2003; Rajbhandari and Piya, 2005). Since these defects affect the quality and quantity of life, treatment of diabetic neuropathy or prevention of its accompanying symptoms has been considered as a major goal. Although several therapeutic interventions and various herbal and chemical medications have already been suggested for the prevention of diabetes complications, their exact effectiveness as reliable preventive medications for diabetic neuropathy has not been considered seriously yet. Melatonin is one of the substances that its therapeutic effects on decreasing complications of diabetes have been reported in some studies. In the present study, preventive effects of melatonin on occurrence of complications such as muscle weakness, nerve tissue injury and decrease of MNCV in diabetic rats were observed.

MNCV at the end of the 7th week following diabetes induction had significant decrease $(\mathrm{p}<0.05)$ in the diabetic groups of sham and DMSO compared to the control group. While, i.p. injection of melatonin after diabetes induction for 6 weeks prevented diabetes destructive effects on nerve conductivity in sciatic-tibia motor nerve.

Oxidative stresses increase and mitochondrial dysfunction are among pathophysiologic causes in neurodegenerative diseases such as Alzheimer, Parkinson and diabetes-induced peripheral neuropathy and the protective effect of melatonin in some of these neurodegenerative diseases has been proved (Tunez et al.,
2003; Leon et al., 2004; Srinivasan, et al., 2006). Melatonin with regard to its various roles in relation to free radicals scavenging, regulation of oxidant and pro oxidant enzymes and also preventing the formation of mitochondrial radicals has a special importance in the treatment of neurodegenerative diseases (Srinivasan et al., 2006). There are several studies showing induction of oxidative stress due to the increase of free radicals in hyperglycemic conditions (Kumar et al., 2007; Saini et al., 2007). Vascular defect following oxidative stress decreases blood flow and causes hypoxia and decrease of nerve function leading eventually to MNCV reduction (Saini et al., 2007; Yorek et al., 2004).

MNCV decrease can be resulted from the change of nerve fiber diameter or myelin layer defect (Yorek et al., 2002; Azcoitia et al., 2003). In line with study Azcoitia et al. (2003) and Yorek et al. (2002) study, our study showed that STZ administration increases morphological changes of myelin layer (sheath) in sciatic nerve. MFD and AD in diabetic groups of sham and DMSO showed significant decrease compared to the control group, while melatonin prevented morphological changes of sciatic nerve in diabetic rats. Sciatic nerve injury associated with morphologic changes was confirmed by explorative behavior reduction and decrease of motor activity in Open field Test, while melatonin administration following decrease of tissue changes prevented significant changes of explorative behavior in diabetic rats. Zencirci et al. (2010) showed that 21 days treatment with 5 and $21 \mathrm{mg} \mathrm{kg}^{-1}$ melatonin causes increase in nerve conductivity velocity and decrease of latency in injured sciatic nerve of diabetic rats (Zencirci et al., 2010). Sciatic nerve injury and increase in response to thermal nociception stimulations in diabetic neuropathy reported in several studies can be the reason of rats' motor activity and explorative behavior reduction. Therefore, decrease of sciatic nerve defect following melatonin administration in previous studies and neuroprotective effects of melatonin in regard to sciatic nerve injury in our study can explain motor activity improvement in diabetic rats following melatonin administration. Some studies have reported that MNCV decrease in diabetes is due to alterations of Na-K ATPase pump activity and sodium gradient (Gerbi et al., 1999; Negi et al., 2010). Alteration of Na-K pump activity can be attributed to the tissue injuries and metabolism abnormality following hyperglycemia in diabetic patients. Decrease of nerve blood flow in diabetic neuropathy leads to nerve metabolic abnormality and consequently defect of ATP-sensitive ion exchanger pumps like $\mathrm{Na}-\mathrm{K}$ pump. Defect of Na-k pump is finally led to the membrane disability in preserving resting potential and consequently disturbs nerve conductivity (Negi et al., 
2010; Gerbi et al., 1999). There are several studies indicating beneficial effects of some medicines and herbal compounds in the improvement of Na-K pump activity and consequently returning $\mathrm{MNCV}$ into the normal rate. Oner et al. (2002) have reported that pretreatment with $10 \mathrm{mg} \mathrm{kg}^{-1}$ melatonin prior to the administration of ethanol prevents ethanol-induced $\mathrm{Na}$ $\mathrm{K}$ ATPase and $\mathrm{Ca}_{2}^{+}$-ATPase inhibition in rat synaptozomes and causes neuroprotection against harmful effects of ethanol through this mechanism (Oner et al., 2002). In human red blood cells, too, the moderating role of melatonin on Na-K ATPase activity and $\mathrm{Na}^{+}-\mathrm{H}^{+}$exchanger has been recognized. This fact is related to the antioxidant effects and moderating role of melatonin on membrane fluidity (Bhatti et al., 2011).

Peripheral neuropathy in primary stages is associated with elevation of nerve fiber activity and disturbs normal sensitivity of neurotic system to injuring and painful stimuli resulting in diabetic hyperalgesia (Malcangio and Tomlinson, 1998; Watson et al., 2003). In the present study, too, in the sham group response time to thermal pain had significant decrease compared to the control group and caused a kind of diabetic hyperalgesia to the thermal pain, while DMSO as melatonin solution showed better protective

effects in Hot Plate Test and caused an increase in response time to thermal pain compared to the sham and melatonin groups. Similar to the present study, Colucci $e t$ al (2008) have asserted that DMSO has antinociceptive effects against thermal pain in Hot Plate and Tail Flick Tests. It seems that in studies that use DMSO as solution, these effects in relation to the characteristics of the administered medicines should be considered.

\section{CONCLUSION}

Therefore, it seems that melatonin administration in early stages of diabetes induction prior to the neurotic damage and occurrence of diabetic neuropathy can decrease the destructive progress of diabetes and causes neuroprotection against damages resulting from diabetic hyperglycemia. Melatonin probably exerts its protective effects through several specified routes such as decrease of free radicals production, Cyclooxygenase inhibition, and moderating role on Na-K AT Pase pump and also activating opioids receptors.

\section{ACKNOWLEDGEMENT}

The present manuscript is the product of a research project that was approved and supported by the Neuroscience Research Center in Kerman University of Medical Science.

\section{REFERENCES}

Arora, M., A. Kumar, R.K. Kaundal and S.S. Sharma, 2008. Amelioration of neurological and biochemical deficits by peroxynitrite decomposition catalysts in experimental diabetic neuropathy. Eur. J. Pharmacol., 596: 77-83. DOI: 10.1016/j.ejphar.2008.08.003

Arreola-Espino, R., H. Urquiza-Marín, M. AmbrizTututi, C.I. Araiza-Saldana and N.L. RochaGonzalez et al., 2007. Melatonin reduces formalininduced nociception and tactile allodynia in diabetic rats. Eur. J. Pharmacol., 577: 203-210. DOI: 10.1016/j.ejphar.2007.09.006

Azcoitia, I., E. Leonelli, V. Magnaghi, S. Veiga and L.M. Garcia-Segura et al., 2003. Progesterone and its derivatives dihydroprogesterone and tetrahydroprogesterone reduce myelin fiber morphological abnormalities and myelin fiber loss in the sciatic nerve of aged rats. Neurobiol. Aging, 24: 853-860. DOI: 10.1016/S0197-4580(02)00234-8

Bhatti, J.S., I.P.S. Sidhu and G.K. Bhatti, 2011. Ameliorative action of melatonin on oxidative damage induced by atrazine toxicity in rat erythrocytes. Molecular Cellular Biochem., 253: 139-149. DOI: 10.1007/s11010-011-0780-y

Geoffriau, M., J. Brun, G. Chazot and B. Claustrat, 1998. The physiology and pharmacology of melatonin in humans. Horm. Res., 49: 136-141. DOI: $10.1159 / 000023160$

Gerbi, A., J.M. Maixent, J.L. Ansaldi, M. Pierlovisi and T. Coste et al., 1999. Fish oil supplementation prevents diabetes-induced nerve conduction velocity and neuroanatomical changes in rats. J. Nutr., 129: 207-213. PMID: 9915901

Golombek, D.A., P. Pevet and D.P. Cardinali, 1996. Melatonin effects on behavior: possible mediation by the central GABAergic system. Neurosci. Biobehav. Rev., 20: 403-412. DOI: 10.1016/01497634(95)00052-6

Gutierrez-Cuesta, J., F.X. Sureda, M. Romeu, A.M. Canudas and B. Caballero et al., 2007. Chronic administration of melatonin reduces cerebral injury biomarkers in SAMP8. J. Pineal Res., 42: 394-402. DOI: 10.1111/j.1600-079X.2007.00433.x

Hardeland, R. and S. Pandi-Perumal, 2005. Melatonin, a potent agent in antioxidative defense: Actions as a natural food constituent, gastrointestinal factor, drug and prodrug. Nutr. Metab., 2: 22-22. PMID: 16153306

Kumar, A., R.K. Kaundal, S. Iyer and S.S. Sharma, 2007. Effects of resveratrol on nerve functions, oxidative stress and DNA fragmentation in experimental diabetic neuropathy. Life Sci., 80: 1236-1244. DOI: 10.1016/j.lfs.2006.12.036 
Leon, J., D. Acuna-Castroviejo, R.M. Sainz, J.C. Mayo and D.X. Tan et al., 2004. Melatonin and mitochondrial function. Life Sci., 75: 765-790. DOI: 10.1016/j.lfs.2004.03.003

Liepinsh, E., R. Vilskersts, L. Zvejniece, B. Svalbe and E. Skapare et al., 2009. Protective effects of mildronate in an experimental model of type 2 diabetes in Goto-Kakizaki rats. Brit. J. Pharmacol., 57: 1549-1556. DOI: $10.1111 / \mathrm{j} .1476-$ 5381.2009.00319.x

Malcangio, M. and D.R. Tomlinson, 1998. A pharmacologic analysis of mechanical hyperalgesia in streptozotocin/diabetic rats. Pain, 76: 151-157. DOI: 10.1016/S0304-3959(98)00037-2

Mantovani, M., R. Pértile, J.B. Calixto, A.R.S. Santos and A.L.S. Rodrigues, 2003. Melatonin exerts an antidepressant-like effect in the tail suspension test in mice: Evidence for involvement of N-methyl-daspartate receptors and the 1-arginine-nitric oxide pathway. Neurosci. Lett., 343: 1-4. DOI: 10.1016/S0304-3940(03)00306-9

Negi, G., A. Kumar, R.K. Kaundal, A. Gulati and S.S. Sharma, 2010. Functional and biochemical evidence indicating beneficial effect of Melatonin and Nicotinamide alone and in combination in experimental diabetic neuropathy. Neuropharmacology, 58: 585-592. DOI: 10.1016/j.neuropharm.2009.11.018

Oner, P., F. Cinar, H. Kocak and F. Gurdol, 2002. Effect of exogenous melatonin on ethanol-induced changes in $\mathrm{Na}(+), \mathrm{K}(+)-$ and $\mathrm{Ca}(2+)$-ATPase activities in rat synaptosomes. Neurochem. Res., 27: 1619-1623. PMID: 12515314

Pevet, P., M.G.M. Balemans, W.C. Legerstee and B. Vivien-Roels, 1980. Circadian rhythmicity of the activity of Hydroxyindole-O-Methyl Transferase (HIOMT) in the formation of melatonin and 5methoxytryptophol in the pineal, retina, and harderian gland of the golden hamster. J. Neural Trans., 49: 229-245. DOI: 10.1007/BF01252128

Rajbhandari, S.M. and M.K. Piya, 2005. A brief review on the pathogenesis of human diabetic neuropathy: Observations and Postulations. Int. J. Diabetes Metab., 13: 135-140.

Saini, A.K., H.S.A. Kumar and S.S. Sharma, 2007. Preventive and curative effect of edaravone on nerve functions and oxidative stress in experimental diabetic neuropathy. Eur. J. Pharmacol., 568: 164-172. DOI: 10.1016/j.ejphar.2007.04.016

Schmeichel, A.M., J.D. Schmelzer and P.A. Low, 2003. Oxidative injury and apoptosis of dorsal root ganglion neurons in chronic experimental diabetic neuropathy. Diabetes, 52: 165-171. DOI: 10.2337/diabetes.52.1.165

Shabani, M., N. Hosseinmardi, M. Haghani, V. Shaibani and M. Janahmadi, 2011. Maternal exposure to the CB1 cannabinoid agonist WIN 55212-2 produces robust changes in motor function and intrinsic electrophysiological properties of cerebellar Purkinje neurons in rat offspring. Neuroscience, 172: 139-152. DOI: 10.1016/j.neuroscience.2010.10.031

Sharma, S., S.K. Kulkarni and K. Chopra, 2007. Effect of resveratrol, a polyphenolic phytoalexin, on thermal hyperalgesia in a mouse model of diabetic neuropathic pain. Fundamental Clin. Pharmacol., 21: 89-94. DOI: $10.1111 / \mathrm{j} .1472-$ 8206.2006.00455.x

Sharma, S.S., A. Kumar, M. Arora and R.K. Kaundal, 2009. Neuroprotective potential of combination of resveratrol and 4-amino 1,8 naphthalimide in experimental diabetic neuropathy: Focus on functional, sensorimotor and biochemical changes. Free Radical Res., 43: 400-408. DOI: 10.1080/10715760902801509

Sima, A.A.F., 2003. New insights into the metabolic and molecular basis for diabetic neuropathy. Cellular Molecular Life Sci., 60: 2445-2464. DOI: 10.1007/s00018-003-3084-X

Srinivasan, V., S.R. Pandi-Perumal, D.P. Cardinali, B. Poeggeler and R. Hardeland et al., 2006. Melatonin in Alzheimer's disease and other neurodegenerative disorders. Behav Brain Funct, 2: $1-23$.

Srinivasan, V., S.R. Pandi-Perumal, D.W. Spence, A. Moscovitch and I. Trakht et al., 2010. Potential use of melatonergic drugs in analgesia: Mechanisms of action. Brain Res. Bull., 81: 362-371. DOI: 10.1016/j.brainresbull.2009.12.001

Srivastava, S.K., K.V. Ramana and A. Bhatnagar, 2005. Role of aldose reductase and oxidative damage in diabetes and the consequent potential for therapeutic options. Endocrine Rev., 26: 380-392. DOI: 10.1210/er.2004-0028

Sugimoto, K., M. Yasujima and S. Yagihashi, 2008. Role of advanced glycation end products in diabetic neuropathy. Curr. Pharm. Design, 14: 953961. DOI: $10.2174 / 138161208784139774$

Tan, D.X., L. Manchester, M. Terron, L. Flores and R. Reiter, 2007. One molecule, many derivatives: A never-ending interaction of melatonin with reactive oxygen and nitrogen species? J. Pineal Res., 42: 28-42. DOI: 10.1111/j.1600-079X.2006.00407.x 
Tunez, I., M.D.C. Munoz, M. Feijóo, J.R. Munoz Castaneda and I. Bujalance et al., 2003. Protective melatonin effect on oxidative stress induced by okadaic acid into rat brain. J.Pineal Res., 34: 265268. DOI: 10.1034/j.1600-079X.2003.00039.x

Watson, C.P.N., D. Moulin, J. Watt-Watson, A. Gordon and J. Eisenhoffer, 2003. Controlled-release oxycodone relieves neuropathic pain: a randomized controlled trial in painful diabetic neuropathy. Pain, 105: 71-78. DOI: 10.1016/S03043959(03)00160-X

Yagihashi, S., S.I. Yamagishi and R. Wada, 2007. Pathology and pathogenetic mechanisms of diabetic neuropathy: Correlation with clinical signs and symptoms. Diabetes Res. Clin. Practice, 77: S184-S89. DOI: 10.1016/j.diabres.2007.01.054

Yamagishi, S.I., S. Ogasawara, H. Mizukami, N. Yajima and R. Wada et al. 2008. Correction of protein kinase $\mathrm{C}$ activity and macrophage migration in peripheral nerve by pioglitazone, peroxisome proliferator activated- $\boldsymbol{\gamma}$-ligand, in insulin-deficient diabetic rats. J. Neurochem., 104: 491-499. DOI: 10.1111/j.1471-4159.2007.05050.x
Yorek, M.A., L.J. Coppey, J.S. Gellett, E.P. Davidson and X. Bing et al., 2002. Effect of treatment of diabetic rats with dehydroepiandrosterone on vascular and neural function. Am. J. Phys. Endocrinol. Metab., 283: E1067-E1075. DOI: 10.1152/ajpendo.00173.2002

Yorek, M.A., L.J. Coppey, JS.. Gellett and E.P. Davidson, 2004. Sensory nerve innervation of epineurial arterioles of the sciatic nerve containing calcitonin gene-related peptide: Effect of streptozotocin-induced diabetes. Exp. Diab. Res., 5: $187-193$.

Zangiabadi, N. M.N. Ahrari and N. Nakhaee, 2007. The effect of omega-3 fatty acids on Nerve Conduction Velocity (NCV) and F-wave latency in patients with diabetic polyneuropathy. Am. J. Pharmacol. Toxicol., 2: 1-3. DOI: 10.3844/ajptsp.2007.1.3

Zencirci, S.G., M.D. Bilgin and H. Yaraneri, 2010. Electrophysiological and theoretical analysis of melatonin in peripheral nerve crush injury. J. Neurosci. Methods, 191: 277-282. DOI: 10.1016/j.jneumeth.2010.07.008 